\title{
Review of: "Exposure of infants to organochlorine pesticides from breast milk consumption in southwestern Ethiopia"
}

\author{
Ayhan Filazi ${ }^{1}$ \\ 1 Ankara University
}

Potential competing interests: The author(s) declared that no potential competing interests exist.

This study was conducted to determine the level of organochlorine pesticides in human breast milk collected from three districts of southwestern Ethiopia at three times points baselines (1st month), midline (six months), and end line (12 months) and risk of infants' exposure. It is really important work and gives important information. However, the most important deficiency is that the milk samples used were collected 5 years ago. No matter how well you store these milk samples, I do not think it will reflect the results correctly. In addition, although it was stated how the method was validated, the validation results were not given. The internal standard was not used or mentioned.

It was stated that $p, p^{\prime}-D D E$ and $p, p^{\prime}-D D T$ were found in all milks. However, GC analyzes also show fatty acids in milk. How can you be sure that DDE or DDT does not mix with the fatty acids in milk? Most of the time, the peak of fatty acids and the DDE or DDT peak are seen at the same time. I wish you would have given a picture of the peaks.

You said that as was difficult to get blank breast milk, the validation test was done using baby formula milk as a blank sample. However, these matrices are different. I wish you could do it with a breast milk. Thus, a comparison with the spike rate could be made. This is always possible.

In section of Instrument analysis of the samples. You mentioned deltamethrin (For deltamethrin determination). Please explain, have you done a deltamethrin analysis? Did you analyze both organochlorine and deltamethrin at the same time?

You did not provide the LOq and LOD results. What value did you use for the samples that you could not detect p,p-DDD and o,p-DDT while performing statistics? Zero?

Figures 2 and 3 not fully understood. How were the results handled? Wouldn't it be more accurate if you did the Spearman test?

It is not clear how you compare in Table 2 . What does c represent?

What is to be understood from Table 3 ? 